Electronic version of an article published as [Nuclear instruments and methods in physics research, 1984, vol.223, No.1, p. 166-172] [DOI: doi:10.1016/0167-5087(84)90262-X]

(C) [copyright Elsevier] 


\title{
HEAVY ION ISOTOPE RESOLUTION WITH POLYMER DETECTORS
}

\author{
A. VIDAL-QUADRAS, M. ORTEGA, F. FERNÁNDEZ, J.L. FONT, M. CASAS, \\ C. BAIXERAS and M. GONZALO * \\ Laboratorio de Fisica Corpuscular, Departamento de Fisica Fundamental, Universidad Autónoma de Barcelona, \\ Bellaterra (Barcelona), Spain
}

Received 14 February 1983 and in revised form 1 November 1983

The heavy ion mass resolution power of polymer detectors Lexan and cellulose nitrate is systematically studied both for accelerator and for cosmic ions. It is concluded that a satisfactory isotopic discrimination, better than $1 \mathrm{u}$, is hardly attainable with these detectors.

\section{Introduction}

The study of the isotopic composition of heavy cosmic rays gives essential information on nucleosynthesis processes [1]. Isotopic abundances are indicators of clue parameters in nucleosynthesis calculations, such as temperature and density. Moreover, most of the difficulties inherent to the examination of elemental ratios can be eliminated or considerably reduced if instead of analyzing a wide charge spectrum, one concentrates on the isotopes of a key element such as carbon, oxygen or iron. Therefore, a considerable effort has been devoted in the last years to study the possibility of heavy ion isotopic discrimination by means of solid state nuclear track detectors [2,3] and several experiments have been performed claiming mass resolutions better than $1 \mathrm{u}$ both for the $\mathrm{VH}(20 \leq Z \leq 28)$ and $\mathrm{M}(6 \leq Z \leq 10)$ groups.

Isotopic ratios for cosmic iron have been measured using Lexan polycarbonate, either UV-sensitized [4] or non-sensitized [5,6]. As for the $\mathrm{M}$ group, cellulose nitrate has been used to measure isotopic ratios in the charge interval $5 \leq Z \leq 8[7-10]$.

It has been stated $[2,3]$ that plastic detectors can compete advantageously with electronic detectors in the iron region and beyond, and optimistic values for mass resolutions of polymer detectors have been predicted [2].

In this paper, systematic studies of the mass resolving power of Lexan polycarbonate for iron and cellulose nitrate for carbon and oxygen are presented. Results are given for cosmic and also for accelerator ions.

Some of the material herein has been previously

* Present address: Servicio de Informática, Fuerzas Eléctricas de Cataluña S.A., Barcelona, Spain. communicated at the 10th and 11th International Conference on Solid state nuclear track detectors held in July 1979 in Lyon [11] and in September 1981 in Bristol [12-14], respectively.

\section{Charge and mass discrimination method based on the} relation etch rate-energy loss

\subsection{Description of the method}

The determination of charge and mass numbers $(Z, A)$ for each registered track in the detector is based on the relation existing between energy loss $\mathrm{d} E / \mathrm{d} x$ and residual range $R$ along the heavy ion path. In the case of a polymer detector, such as Lexan or $C N$, the parameter closely related to $\mathrm{d} E / \mathrm{d} x$ in a certain point of the track is the etch-rate $V_{\mathrm{T}}$ in this point [15] according to the functional dependence of $V_{\mathrm{T}}$ on $\mathrm{d} E / \mathrm{d} x, V_{\mathrm{T}}=$ $f(\mathrm{~d} E / \mathrm{d} x)$. Each track is so characterized by a set of experimental $\left(V_{\mathrm{T}}, R\right)$ points measured along its registration range and a one-to-one correspondence should exist between the set of $(Z, A)$ pairs and the set of $V_{\mathrm{T}}-R$ curves on a $V_{\mathrm{T}}-R$ plot.

The geometry of multi-cone tracks and the measurement of their geometrical parameters have been abundantly described [15-17], as well as the procedure to calculate experimental uncertainties in etch rate, $\Delta V_{\mathrm{T}}$, and in residual range, $\Delta R$.

Several models have been tried in order to predict quantitatively the registration threshold and etch-rate along a heavy ion track in a solid. Total energy loss, secondary energy loss, restricted energy loss and primary ionization have been considered to govern track formation $[15,18,19]$.

In this paper both primary ionization and restricted 
energy loss criterions have been applied. In any case, the number of free parameters to be fitted in the relation $V_{\mathrm{T}}=f(\mathrm{~d} E / \mathrm{d} x)$ is three, and charge and mass spectra are not affected by the use of one or another criterion.

The function $V_{\mathrm{T}}=f(\mathrm{~d} E / \mathrm{d} x)$ that best reproduces the experimental behaviour for most plastic detectors if of the power-like type $[14,15]$ :

$V_{\mathrm{T}}=B(\mathrm{~d} E / \mathrm{d} x)^{n}$,

where $B$ and $n$ are to be obtained from a least-squares fit to the experimental $\left(V_{\mathrm{T}}, R\right)$ points belonging to a well known calibration ion.

The dependence on the mass number $A$ is given through the velocity $\beta$ by the range-energy relation in the particular polymer used. The method due to Henke and Benton [20] has been applied to $\mathrm{CN}$ and to Lexan in order to obtain the range-energy curves for the different ions.

When the restricted energy loss (REL) criterion is adopted [15], the three parameters to be fitted in eq. (1) are $B, n$ and the threshold energy $E_{0}$ marking the division line between close and distant collisions. Once these parameters are determined by means of a chi-square minimization [21], a "theoretical" relation $V_{\mathrm{T}}=f[\mathrm{~d} E(R, Z, A) / \mathrm{d} x]$ is available as discrimination tool. Uncertainties in $Z, \Delta Z$, and in $A, \Delta A$, are estimated varying the minimum chi-square value in one unit [11].

\subsection{Isotopic resolution for iron with Lexan}

The validity and limitations of the method outlined will be first illustrated by its application to 183 cosmic tracks registered in Lexan with a mean energy of 250 $\mathrm{MeV} / \mathrm{nuc}$ at the detector level. The composition of the stack and exposure conditions have already been published [16].

The highest frequency points on a two-dimensional $V_{\mathrm{T}}-R$ plot are considered to be $Z=26$, iron being the most abundant ion in the $\mathrm{VH}$ group of primary cosmic radiation. A third-order polynomial is fitted to these points and an error band fixed between limits corresponding to vary in $\pm 10 \%$ the zero-order coefficient of the polynomial, according to the average experimental uncertainty in an etch-rate measurement. All tracks with more than $75 \%$ of their points inside the band are supposed to be calibration ions $Z=26$. Fifteen tracks are selected in this way from the total 183, giving 138 $\left(V_{\mathrm{T}}, R\right)$ calibration points.

The REL criterion is adopted and a chi-square minimization leads to the values for the parameters $B$ and $n$. The threshold energy is chosen as $E_{0}=1000 \mathrm{eV}$, in agreement with the literature $[2,4]$.

The resolution power of the detector for the $\mathrm{VH}$ group will depend on the sensitivity of the restricted energy loss $\mathrm{REL}_{1000}$ to variation is in charge and mass number of the ion considered. The relation $V_{\mathrm{T}}(R, Z, A)=B\left[\operatorname{REL}\left(R, Z, E_{0}\right)\right]^{n}$, with the values for $B, n$ and $E_{0}$ furnished by the chi-square minimization, permits to draw the $V_{\mathrm{T}}-R$ curves corresponding to charge numbers $20<Z<28$, as well as the $V_{\mathrm{T}}-R$ curves for $Z=26$ and iron's stable isotopes $A=54,56$ and 58 .

The comparison of etch-rate differences between adjacent charge and mass numbers to the experimental uncertainty $\Delta V_{\mathrm{T}}$ is essential to appraise the discrimination power of Lexan for the $\mathrm{VH}$ group.

Representative etch-rate values and their differences for adjacent charges $Z=25,26$ and 27 are given in table 1 at residual ranges 500 and $1000 \mu \mathrm{m}$. A feeling for the sensitivity of the etch-rate function to mass variations can also be obtained from table 1 . The etch-rate differences in table 1 should be compared to the experimental uncertainty $\Delta V_{\mathrm{T}}$. The distribution of $\Delta V_{\mathrm{T}}$ values is shown in fig. 1, leading to an average value $\left\langle\Delta V_{\mathrm{T}}\right\rangle=$ $0.09 \mu \mathrm{m} / \mathrm{h}$.

Distributions of charge and mass uncertainties $\Delta Z$ and $\Delta A$ are also displayed in fig. 1 , with average values $\langle\Delta Z\rangle=0.2$ charge units and $\langle\Delta A\rangle=1.2 \mathrm{u}$.

This discrimination method, based on the relation

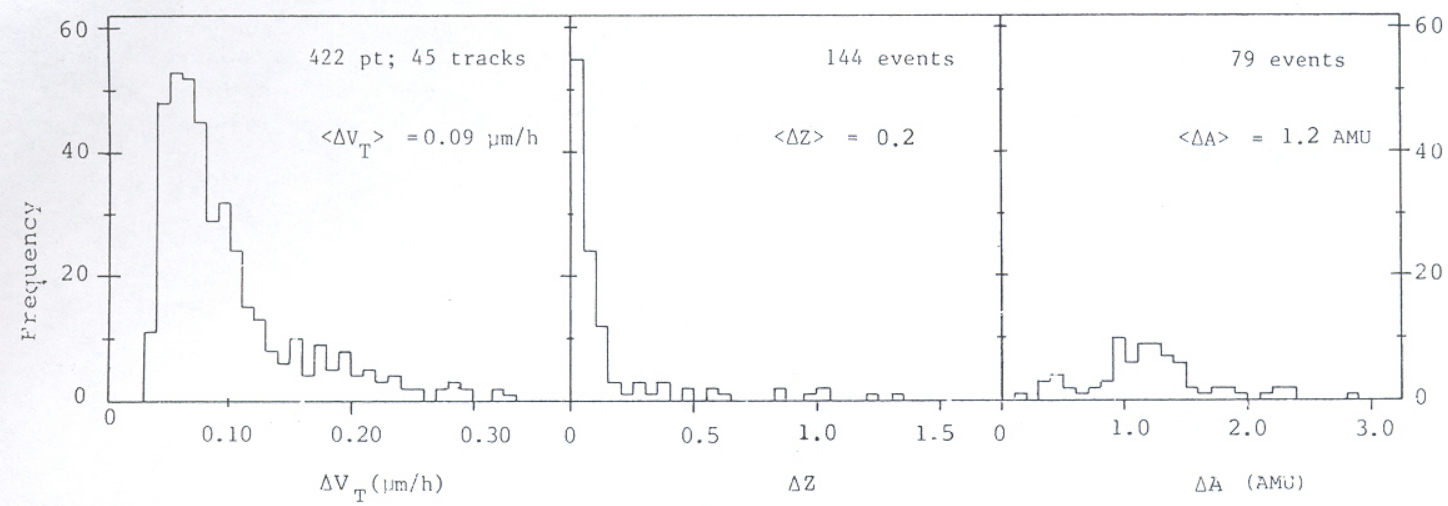

Fig. 1. Etch-rate, charge and mass uncertainties for cosmic VH ions registered in Lexan polycarbonatc, 
Table 1

Etch-rate values and etch-rate differences in Lexan polycarbonate calculated according to relation (3) for (a) cosmic iron calibration an (b) accelerator iron calibration

\begin{tabular}{|c|c|c|c|c|}
\hline$R(\mu \mathrm{m})$ & $Z$ & $A$ & $\begin{array}{l}V_{\mathrm{T}} \\
(\mu \mathrm{m} / \mathrm{h})\end{array}$ & $\begin{array}{l}\text { Etch-rate } \\
\text { differences } \\
(\mu \mathrm{m} / \mathrm{h})\end{array}$ \\
\hline 500 (a) & $\begin{array}{l}25 \\
26\end{array}$ & $\begin{array}{l}54 \\
56 \\
58\end{array}$ & $\begin{array}{l}1.800 \\
1.934 \\
2.008 \\
2.082 \\
2.311\end{array}$ & $\begin{array}{l}0.208 \\
0.074 \\
0.074 \\
0.304\end{array}$ \\
\hline 1000 (a) & $\begin{array}{l}25 \\
26 \\
\\
27\end{array}$ & $\begin{array}{l}54 \\
56 \\
58\end{array}$ & $\begin{array}{l}0.873 \\
0.940 \\
0.976 \\
1.013 \\
1.127\end{array}$ & $\begin{array}{l}0.103 \\
0.036 \\
0.037 \\
0.151\end{array}$ \\
\hline $500(b)$ & $\begin{array}{l}25 \\
26 \\
27\end{array}$ & & $\begin{array}{l}1.676 \\
1.820 \\
2.039\end{array}$ & $\begin{array}{l}0.144 \\
0.219\end{array}$ \\
\hline 1000 (b) & $\begin{array}{l}25 \\
26 \\
27\end{array}$ & & $\begin{array}{l}0.883 \\
0.960 \\
1.078\end{array}$ & $\begin{array}{l}0.077 \\
0.117\end{array}$ \\
\hline
\end{tabular}

between etch-rate and energy loss, has also been applied to 22 iron ions of $150 \mathrm{MeV} /$ nuc energy from the Berkeley Bevalac. A stack of 40 Lexan layers of $250 \mu \mathrm{m}$ thickness and $40 \mathrm{~cm}^{2}$ surface area was exposed to the Berkeley Bevalac iron beam at $45^{\circ}$ incidence angle. Etching conditions were the same as applied to cosmic ions and have been described elsewhere [16].

In this case, the primary ionization criterion [15] was adopted. Once the discrimination procedure is carried out, the charge and mass spectra appear as in fig. 2 . Average values for experimental uncertainties are $\left\langle\Delta V_{\mathrm{T}}\right\rangle=0.08 \mu \mathrm{m} / \mathrm{h},\langle\Delta Z\rangle=0.2$ charge units and

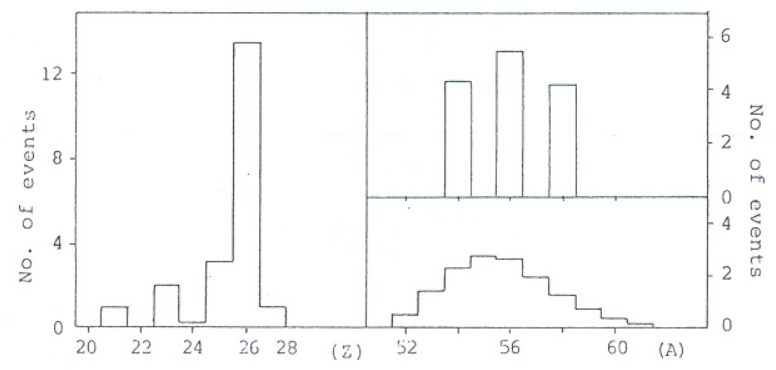

Fig. 2. Charge and mass spectra for 22 iron ions of 150 $\mathrm{MeV} /$ nuc stopping in Lexan polycarbonate. The few ions $Z<26$ are probably due to impurities in the beam or to fragmentation. The poverty of mass resolution is clearly shown. In the upper mass spectrum, only $A=54,56$ and 58 have been imposed as possible mass numbers.
$\langle\Delta A\rangle=1.3$ u. Etch-rate values and differences in etch-rate for adjacent charges are shown in table 1. The slight discrepancy between etch-rate values for cosmic iron or accelerator iron calibration comes from the fact that different Lexan batches have been used in the two experiments and they have not been etched simultaneously.

\subsection{Isotopic resolution for carbon and oxygen with cel- lulose nitrate}

Now the results concerning the study of the $\mathrm{M}$ group with $\mathrm{CN}$ will be exposed. Two stacks of 285 Kodak-CN layers of $210 \mu \mathrm{m}$ thickness and $40 \mathrm{~cm}^{2}$ surface area were exposed to the Berkeley Bevalac ${ }^{16} \mathrm{O}$ and ${ }^{12} \mathrm{C}$ beams at $250 \mathrm{MeV} /$ nuc energy and $45^{\circ}$ incidence angle. Tracks were revealed in the last six layers according to the registration threshold, which is about $20 \mathrm{MeV} /$ nuc for the $\mathrm{M}$ group in $\mathrm{CN}$. The etchant was $6.25 \mathrm{~N} \mathrm{NaOH}$ solution at a temperature of $40.0 \pm 0.1{ }^{\circ} \mathrm{C}$ and the etching time was $5 \mathrm{~h}$. The REL criterion has been used and applied to 26 oxygen tracks and 30 carbon tracks. Two sets of $V_{\mathrm{T}}-R$ curves for the charge interval $5 \leq Z \leq 9$ are shown in fig. 3. One set has been obtained using oxygen as calibration ion and the other by means of carbon calibration. The resulting two sets of curves are so close that they are reduced to one on the graph. Some representative experimental points have been included for illustrative purposes. Distributions of etchrate uncertainty $\Delta V_{\mathrm{T}}$ for carbon and oxygen tracks give in both cases an average value $\left\langle\Delta V_{\mathrm{T}}\right\rangle=1.1 \mu \mathrm{m} / \mathrm{h}$. Uncertainties in charge number $\Delta Z$ are very small,

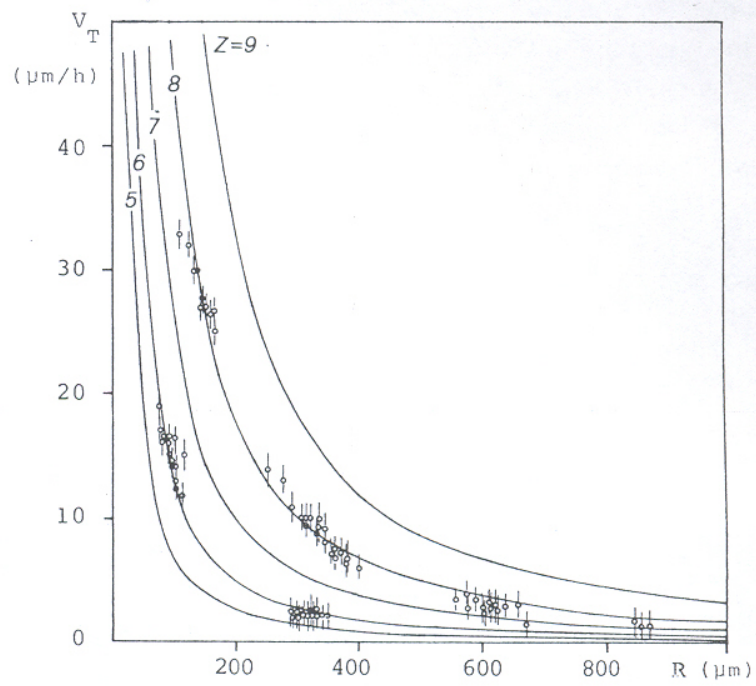

Fig. 3. $V_{\mathrm{T}}-R$ curves for charges $5 \leq Z \leq 9$ in Kodak cellulose nitrate based on oxygen calibration and on carbon calibration. The consistency of both charge scales is such that the shift between both sets of curves cannot be appreciated on the graph. 
$\Delta Z<0.1$ charge units for all identified tracks. Uncertainties in mass number $\Delta A$ give average values $\langle\Delta A\rangle$ $=0.8 \mathrm{u}$ for carbon tracks and $\langle\Delta A\rangle=0.7 \mathrm{u}$ for oxygen tracks.

\section{Charge and mass discrimination method based on mean residual range}

\subsection{Description of the method}

Charge and mass discrimination can also be based on the reduction of all the information in each track to one single parameter, namely mean residual range [4]. A track is characterized by a set of points $(L, R)$, where $L$ stands for cone length and $R$ is the residual range, and a polynomial $L(R)$ can be fitted to each separate track. The polynomial coefficients $A_{k} ; k=0,1 \ldots N$, are taken as free parameters in the fit. $N$ is the smaller nearest integer to half the number of cones in the track. Once a particular set of coefficient values has been assigned to each track, a mean residual range $\bar{R}$ can be defined for it [4]. The peak in the distribution of experimental $\bar{R}$ values can be attributed to the calibration ion and the tracks contained in this peak taken as calibration tracks.

In order to use correctly the mean residual range $\bar{R}$ as discrimination parameter, it is important to accurately evaluate the experimental uncertainty $\Delta \bar{R}$. This can be achieved as illustrated in fig. 4. Two fictitious tracks are fitted to "experimental" points shifted from the real (REL) points according to experimental errors $\Delta L$ and $\Delta R$. Differences between mean residual ranges corresponding to the shifted tracks and the real track represent adequately the experimental uncertainty in $\bar{R}, \Delta \bar{R}$. Parameters $B, n$ and $E_{0}$ are fixed by least-squares minimization of the differences between real and "theoretical" etching times.

The knowledge of parameters $B, n$ and $E_{0}$ allows to establish the curves $L-R$ for the different ions and a

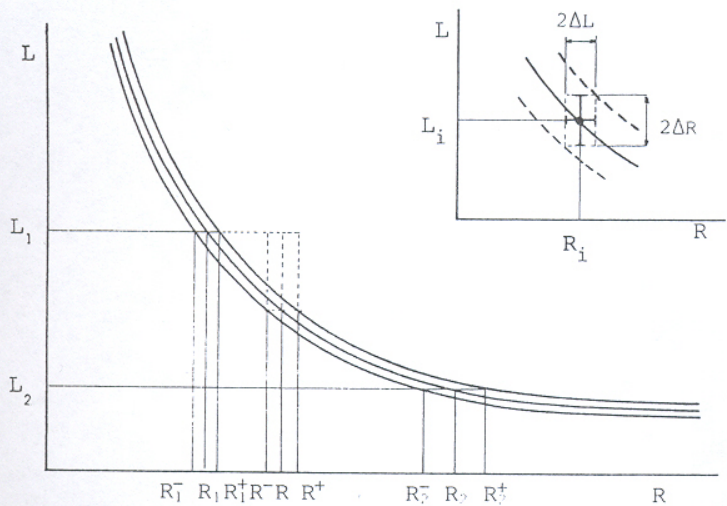

Fig. 4. Estimation of experimental uncertainty in mean residual range $\Delta \bar{R}$.

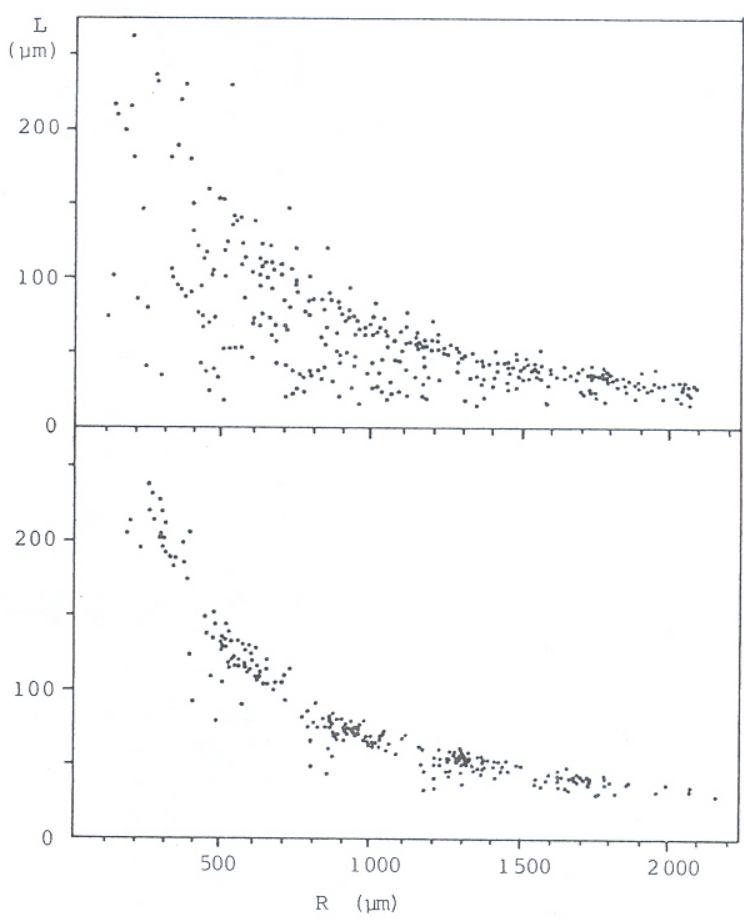

Fig. 5. Experimental $(L, R)$ points for $173 \mathrm{VH}$ ions and for 33 accelerator iron ions registered in Lexan polycarbonate.

charge and mass scale $\bar{R}(Z, A)$ can be settled and experimental distributions of mean residual range superimposed on it, leading to charge, and eventually mass, spectra.

\subsection{Isotopic resolution for iron with Lexan}

Results obtained with cosmic ions will be first exposed. In fig. 5 experimental $(L, R)$ points are displayed corresponding to 173 cosmic ions of 25

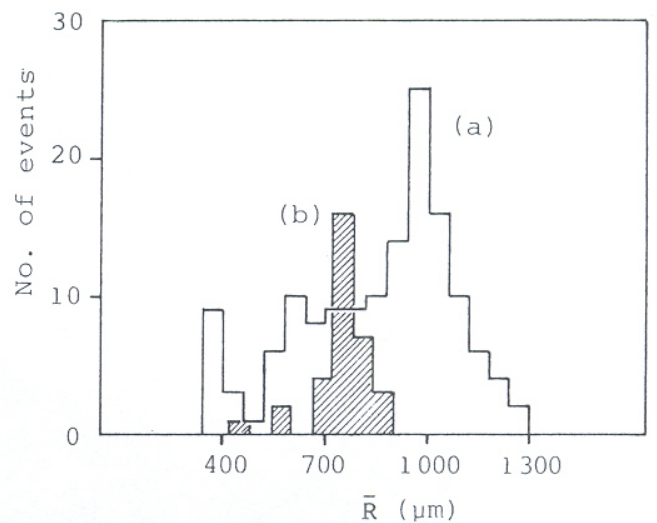

Fig. 6. Distribution of mean residual range values for cosmic $\mathrm{VH}$ ions (a) and for accelerator iron ions (b) registered in Lexan polycarbonate. 
Table 2

Values for the mean residual range $\bar{R}$ and coefficients $A_{k}$ for two representative iron tracks: (a) cosmic and (b) accelerator. Constants $C$ and $\alpha$ in eq. (11) are $C=0$ and $\alpha=1.8$. Cone length $L(R)$ and residual range $R$ in eq. (11) must be expressed in mm in order to use the $A_{k}$ values displayed in table 3 .

\begin{tabular}{|c|c|c|c|c|c|}
\hline $\bar{R}(\mu \mathrm{m})$ & $A_{0}$ & $A_{1}$ & $A_{2}$ & $A_{3}$ & $A_{4}$ \\
\hline (a) $\begin{array}{l}\bar{R}_{+}=849 \\
\bar{R}=817 \\
\bar{R}_{-}=785\end{array}$ & $\begin{array}{l}-0.012138 \\
-0.014185 \\
-0.016797\end{array}$ & $\begin{array}{l}0.063939 \\
0.063978 \\
0.063751\end{array}$ & $\begin{array}{l}-0.029149 \\
-0.027948 \\
-0.026535\end{array}$ & $\begin{array}{l}-0.008417 \\
-0.008001 \\
-0.007423\end{array}$ & \\
\hline (b) $\begin{array}{l}\bar{R}_{+}=804 \\
\bar{R}=771 \\
\bar{R}_{-}=739\end{array}$ & $\begin{array}{l}0.043794 \\
0.041238 \\
0.038648\end{array}$ & $\begin{array}{l}-0.049806 \\
-0.051166 \\
-0.052416\end{array}$ & $\begin{array}{l}-0.589401 \\
-0.589311 \\
-0.588611\end{array}$ & $\begin{array}{l}-0.384412 \\
-0.387740 \\
-0.390046\end{array}$ & $\begin{array}{l}-0.089654 \\
-0.091371 \\
-0.092641\end{array}$ \\
\hline
\end{tabular}

Table 3

Values of mean residual ranges defining a charge scale in Lexan polycarbonate. Stable isotopes of iron are also indicated.

\begin{tabular}{|c|c|c|c|c|c|c|c|c|c|c|c|}
\hline$Z$ & 20 & 21 & 22 & 23 & 24 & 25 & & 26 & & 27 & 28 \\
\hline A & & & & & & & 54 & 56 & 58 & & \\
\hline $\bar{R}(\mu \mathrm{m})$ & 386 & 483 & 571 & 670 & 752 & 871 & 938 & 972 & 1007 & 1113 & 1191 \\
\hline
\end{tabular}

$\mathrm{MeVC} /$ nuc average energy at detector level and registered in Lexan polycarbonate. In table 2 , the values of the coefficients $A_{k}$ of the $L(R)$ polynomial and mean residual ranges are given for a representative track and its associated shifted tracks according to experimental errors. It can be seen that $\Delta \bar{R} \simeq 30 \mu \mathrm{m}$. The distribution of mean residual range values is shown in fig. 6 . Tracks included in the outstanding peak are considered to be ${ }^{56} \mathrm{Fe}$. The mean residual ranges defining the charge scale in the interval $20 \leq Z \leq 28$ can be seen in table 3 . Charge spectra derived from the method described in sect. 2.1 and from the one presently considered are shown in fig. 7. It is clear from table 3 that the charge resolution is $\Delta Z \simeq 0.3$ charge units. As for the mass

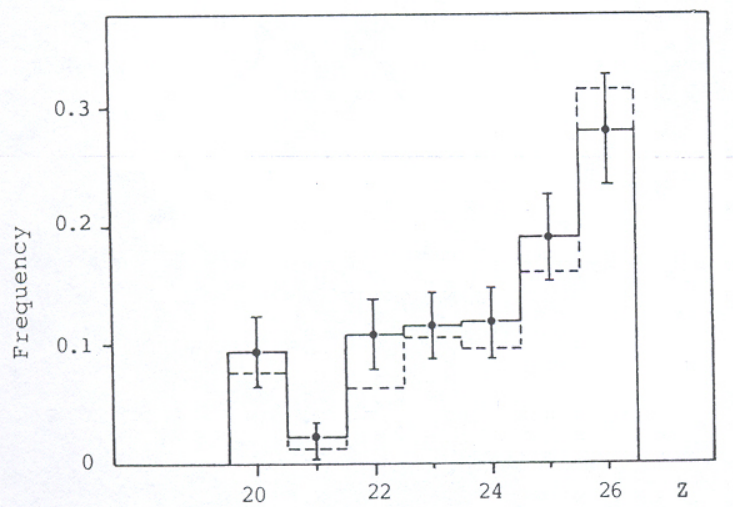

Fig. 7. Cosmic VH group normalized charge spectra calculated according to the methods described in sect. 2.1 and sect. 3.1 for ions registered in Lexan polycarbonate. Agreement is excellent, showing the intrinsic character of estimated resolution power. resolution, the mean residual ranges associated to stable isotopes of iron are included in table 3. According to the differences between then and to the value of the mean residual range uncertainty $\Delta R \simeq 30 \mu \mathrm{m}$, it can be concluded that $\Delta A \simeq 2 \mathrm{u}$ for cosmic iron tracks registered in Lexan.

Discrimination based on the mean residual range has also been applied to 33 accelerator iron ions of 150 $\mathrm{MeV} /$ nuc energy registered in Lexan whose exposure conditions have already been described in sect. 2.2. The experimental $(L, R)$ points are shown in fig. 5 . The distribution of mean residual range values is given in fig. 6. The values of the coefficients $A_{k}$ of the $L(R)$ polynomial and mean residual ranges for a representative track and associated shifted tracks according to experimental errors are given in table 2. Clearly, $\Delta R \simeq 30$ $\mu \mathrm{m}$, the same uncertainty as for cosmic ions. Charge and mass scales can be compared to the uncertainty $\Delta \bar{R}$ in fig. 8, leading to a visualization of charge solution $\Delta Z \simeq 0.3$ charge units and of mass resolution $\Delta A \simeq 2 \mathrm{u}$, in total agreement with the results for cosmic tracks.

\subsection{Isotopic resolution for oxygen with cellulose nitrate}

In this section results concerning 26 multi-cone tracks produced in a Kodak-CN stack by a $250 \mathrm{MeV} /$ nuc

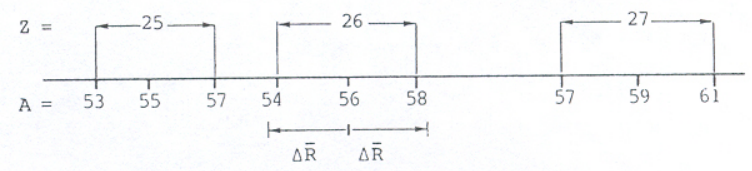

Fig. 8. Charge and mass scales based on mean residual range for Lexan polycarbonate. 

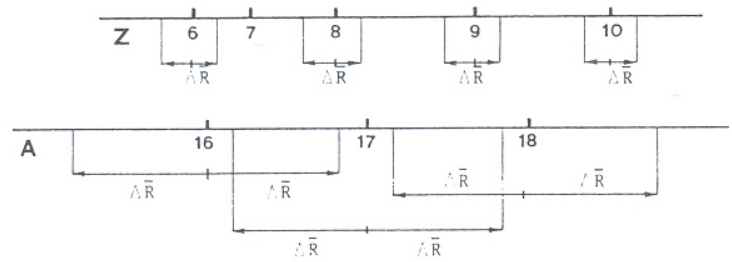

Fig. 9. Charge and mass scales based on mean residual range in Kodak cellulose nitrate.

oxygen beam will be exposed. Details on exposure conditions have already been given in sect. 2.3. The experimental uncertainty in mean residual range happens to be $\Delta \bar{R} \simeq 15 \mu \mathrm{m}$. The resulting charge and mass scales are presented in fig. 9 , leading to a charge resolution in the range $\Delta Z \simeq 0.2-0.4$ charge units and to a mass resolution $\Delta A \simeq 0.8 \mathrm{u}$.

\section{Discussion of results}

It is clear that a good mass resolution for iron ions stopping in Lexan polycarbonate would require the measurement of etch-rate values with an experimental uncertainty better than 3\%, an achievement that, as fig. 1 shows, is far from the possibilities of the detector.

As for the M-group isotopic discrimination with cellulose nitrate, similar conclusions can be reached. It is interesting to point out the consistency of charge scales based on different calibration ions evidenced in fig. 3.

Discrimination based on mean residual range confirms these results indicating a mass resolution of $2 \mathrm{u}$ for iron ions registered in Lexan and of near $1 \mathrm{u}$ for the M-group ions registered in cellulose nitrate.

The agreement between results obtained for cosmic ions and for accelerator ions is remarkable, indicating the validity of accelerator beam calibrations to be applied to the study of cosmic spectra, as some experiments now in progress intend to do [22].

According to what has been shown, Henke and Benton's prediction [2] of $\Delta A \simeq 0.4 \mathrm{u}$ for iron registered in Lexan must be recognized as unrealistic. Neither an incidence angle $\delta=\pi / 2$ nor a value $\rho=0.5$ for the parameter $\rho=2 B / h$, where $B$ is the bulk etch and $h$ the layer thickness before etching, as considered in ref. [2], corresponds to feasible experimental conditions. Introducing our experimental values $\delta=\pi / 4$ and $\rho=0.1$ in relation (4) of ref. [2], a mass resolution $\Delta A \simeq 1 \mathrm{u}$ is estimated. This is consistent with our experimental result $\Delta A \simeq 2 \mathrm{u}$, if the approximate character of Henke and Benton's estimate is kept in mind. Turning to the isotopic resolution $\Delta A \simeq 0.7 \mathrm{u}$ for the iron peak claimed by Siegmon and coworkers [4] with UV-irradiated Lexan, it must be remembered that in ref. [2] a mass resolution $\Delta A \simeq 0.1 \mathrm{u}$ is estimated for this case, that is to say, almost an order of magnitude better, therefore supporting our experimental result for non-irradiated Lexan, well above Henke and Benton's estimate.

With respect to cellulose nitrate mass discrimination for the M group, Henke and Benton's prediction was $\Delta A \simeq 0.4 \mathrm{u}$ for $Z=10$ and a type of cellulose nitrate, the so called USF 3, similar to the one we have been using, manufactured by Kcdak. In ref. [2], a value $\rho=0.32$ is taken for the parameter $\rho=2 B / h$, which, according to our experience, does not correspond to a realistic situation. In our case, $\rho=0.020$, a value of $\rho$ one order of magnitude smaller. A value of $\rho$ one order of magnitude greater would either imply such a long etching time or plates so thin that multi-cone formation, specially for M-group tracks, would be seriously inhibited.

As for a mass resolution $\Delta A \simeq 0.4 \mathrm{u}$ on cellulose nitrate for the $M$ group reported by Beaujean and Enge $[7,8]$ in the $100-200 \mathrm{MeV} /$ nuc region or by Sojka [10] in the $10 \mathrm{MeV} /$ nuc region, this value is difficult to understand when looking at fig. 9 of the present paper.

A significant isotopic resolution of cosmic heavy ions seems out of the scope of the polymer detectors Lexan and cellulose nitrate, and the present trend of the efforts in this important subject is the use of combinations of stacks of these polymers with electronic detectors [23,24]. There is also much hope invested in the high-sensitivity polymer CR-39 [22-25].

The authors want to thank Dr. D. O'Sullivan and Dr. A. Thompson from the Dublin Institute for Advanced Study and Dr. M. Debeauvais from the Centre des Recherches Nucléaires in Strasbourg for their helpful cooperation. Financial support from the Spanish Comisión Nacional de Investigación del Espacio (CONIE), from the Catalan Comissió Interdepartamental de Recerca i Tecnologia (CIRIT), and kind help providing computing facilities from Fuerzas Elétricas de Cataluña (FECSA) are also warmly acknowledged.

\section{References}

[1] S.E. Woosley, Appl. Sp. Sci. 39 (1976) 103.

[2] R.P. Henke and E.V. Benton, Nucl. Track Det. 1 (1977) 93.

[3] W. Enge, Proc. 9th Int. Conf. on Solid state nuclear track detectors, eds., F. Granzer, H. Paretzke and S. Schopper, vol. 2 (Pergamon, Oxford, 1978) p. 1039.

[4] G. Siegmon, K. Barthöloma and W. Enge, ibid., p. 1059.

[5] V.A. Clapham, P.H. Fowler, C. O'Cellaigh, D. O'Sullivan and A. Thompson, Proc. 14th Int. Cosmic ray Conf., Munich, vol. 12 (1975) p. 4128.

[6] R. Scherzer, W. Enge, R. Beaujean, S. Hertzman, S. Kristiansson and K. Söderström, Proc. 10th Conf. on Solid 
state nuclear track detectors, eds., H. François et al. (Pergamon, Oxford, 1980) p. 1063.

[7] R. Beaujean and W. Enge, Z. Physik 256 (1972) 416.

[8] R. Beaujean, H. Sagebiel and W. Enge, Proc. 9th Int. Conf. on Solid state nuclear track detectors, eds., F. Granzer, H. Paretzke and E. Schopper, vol. 2 (Pergamon, Oxford, 1978) p. 1069.

[9] K. Fukui, W. Enge and R. Beaujean, Z. Physik A227 (1976).

[10] B. Sojka, R. Scherzer, H. Rohrs and W. Enge, Proc. 10th Int. Conf. on Solid state nuclear track detectors, eds., $\mathrm{H}$. François et al. (Pergamon, Oxford, 1980) p. 1079.

[11] M. Ortega, A. Vidal-Quadras, F. Fernández, C. Baixeras, M. Casas, M. Gonzalo, J. Medina and J. Sequeiros, ibid., p. 1041.

[12] M. Gonzalo, A. Vidal-Quadras, M. Ortega, F. Fernández, C. Baixeras, M. Casas, J. Sequeiros and J. Medina, Proc. 11 th Int. Conf. on Solid state nuclear track detectors, eds., P.H. Fowler and V.M. Clapham (Pergamon, Oxford, 1982) p. 291.

[13] A. Vidal-Quadras, F. Fernández, M. Gonzalo, M. Ortega, C. Baixeras and M. Casas, ibid., p. 257.

[14] F. Fernández, A. Vidal-Quadras, M. Ortega, C. Baixeras and C. Casas, ibid., p. 261.
[15] R.L. Fleischer, P.B. Price and R.M. Walker, Nuclear tracks in solids (University of California Press, Berkeley, 1975).

[16] J. Sequeiros, J. Medina, A. Durá, M. Ortega, A. VidalQuadras, F. Fernández and R.T. Thorne, Nucl. Instr. and Meth. 135 (1976) 133.

[17] M. Casas, Thesis, Universidad Autónoma de Barcelona (1981).

[18] E.V. Benton, US Naval Defense Research Laboratory Task Report-68-14 (1968).

[19] R.L. Fleischer, Prog. Mat. Sci. Chalmers Anniversary Volume (1981) 97.

[20] E.V. Benton and R.P. Henke, Nucl. Instr. and Meth. 67 (1969) 87.

[21] G.C. Sheppey, CERN D505 (1966).

[22] J.H. Adams, L. Beahm, J.A. Maturi, B. Stiller and A. Thompson, Proc. 11th Int. Conf. on Solid state nuclear track detectors, eds., P.H. Fowler and V.M. Clapham (Pergamon, Oxford, 1982) p. 867.

[23] W. Hunger, G. Siegmon, G. Sermund, R. Beaujean, W. Enge, W.R. Webber and G.A. Simpson, ibid., p. 859.

[24] W. Heinrich, M. Simon, H.O. Tittel, J.F. Ormes, V.K. Balasubrahmanyan and R.E. Streitmatter, ibid., p. 863.

[25] G. Tarlé, S.P. Ahlen and P.B. Price, Proc. 17th Int. Cosmic ray Conf., Paris, vol. 2 (1981) p. 85. 